\title{
Editorial Policy and Instructions for Authors
}

Editorial Policy: In 2004, the Graduate School of Kuroshio Science was established at Kochi University to study the bioresources, environment, and human health conditions in the area under the influence of the Kuroshio, one of the world's largest ocean currents. To achieve our mission, we have been publishing the journal Kuroshio Science twice a year since 2007 so as to cover the integrated arts and sciences, including science, social sciences, and cultural studies related to the Kuroshio area, beyond the borders of any single established field of science. Please visit the URL: http://kuroshio. cc. kochi-u.ac.jp/en/index.html

Editorial board: Editor-in-Chief: Hiroshi Ueda (Kochi University, Japan). Associate Editors: Kazuo Okuda, Yoshiaki liguni, Ichiro Mine, Motomi Kato, Takashi Sugitani, Masayuki Takahashi, Yoshinori Morooka (Kochi University, Japan); Plutomeo M. Nieves (Bicol University, Philippines); Hin-kiu Mok (National Sun Yet-sen University, Taiwan).

Manuscript type: Contributions may take the following manuscript types: Research paper, a peer-reviewed research article having a high academic significance; Research note, a peer-reviewed research article from a preliminary study; Review, a synthesis of research on a topic; Research report, the information on research activities; Essay, Comment, Proposal, and News concerning research, education, or social contribution; and other articles on topics covered by the above policy.

Submission: Submit manuscripts to the Editorial Board of Kuroshio Science, Kochi University (Monobe Otsu 200, Nankoku-shi, 783-8502 Japan; E-mail; km03@kochi-u.ac.jp) in attached files of e-mail or in a CD or DVD disk. For submission of printed or handwritten manuscripts, please consult with the Editor.

Acceptance: The Editorial Board makes the final decisions on acceptance or rejection of manuscripts. Accepted manuscripts are reviewed by the Editorial Board before publication and, if necessary, authors might be required for revision. The types and formats of papers in the journal are left to the Editor's discretion.

Proofs and reprints: The proofreading is done by the author first, and later by the Editorial Board. Fifty reprints are free of charge. Additional reprints can be ordered when returning the author's proofs.

Copyright: The copyright to any published articles in
Kuroshio Science belongs to the Editorial Board of Kuroshio Science, Kochi University.

Reproducing copyright material: If copyright material such as figures published in journals or books is reproduced in the manuscript, a pdf file proving permission from the copyright holder to reproduce the material or a scanned jpg file of written permission should be included when submitted.

Charges: No charges are required for authors. However, costs for printing of color pages or too many print pages might be partly charged to the authors depending on our budget for publication.

Open access: Papers in the journal will appear in pdf files at the journal website (http://kuroshio.cc.kochi-u.ac.jp/repository /en/).

\section{Preparation of manuscript}

1. The manuscript must be written in English or Japanese.

2. The text file should be made as a MS-Word document. The text must be double spaced on A4 sheet size with $30 \mathrm{~mm}$ margins on top, bottom and both sides, and should include the page and line numbers from the first page.

3. The first text page should contain the name of the manuscript type categorized in the Editorial Policy, the title of the paper, the author(s), affiliation(s) of all author(s) along with postal addresses, the e-mail address of the corresponding author (or contact person), and the running title. The title should not exceed 25 words excluding the subtitle, and the running title must not exceed 30 characters. The name(s) of the author(s) must not be abbreviated, and the corresponding author must be marked with a superscript asterisk (*) on the right side of his/her name.

4. In Research papers, Research notes and Review articles, the second page should include an Abstract stating the aims, methodology, results, and conclusions drawn from the study, and Keywords after the Abstract. The length of Abstract must not exceed 250 words. Keywords must not exceed 5 words.

From page 3, the text of science fields generally contains the following sections, Introduction, Materials and Methods, Results, Discussion, (Acknowledgements,) and References. Headings of the sections should be typed in bold face with center alignment. Subheadings within a section should be in bold face and in left alignment.

5. Terminology, including abbreviations and acronyms, should follow the general usage in each field. Type a space between a digit and a unit (e.g. $1 \mathrm{~cm}$ ) except for $\%$ and ${ }^{\circ} \mathrm{C}$. 
Species and genus names of organisms must be in italic type.

6. Figures and tables should be prepared in pdf, Power Point, Excel (for tables), or jpg (for figures) files, or embedded at the end of the text file. For other file types, consult with the Editor.

7. Letter size used in figures should be a readable one if the figures are resized to be printed within a column (ca. 80 $\mathrm{mm}$ ) of the page. Figure and table legends must be provided at the end of the text file and above each table in the table file, respectively.

8. Figures and tables must be numbered as Fig. 1 and Table 1 in order of the first appearance of each one in the text.

9. All references cited must be listed in the References section in alphabetical order of the author name(s) by following the examples. The order of the references by the same author(s) should follow the general rules for academic journals.

1) Journals

Clarke, K.R. 1993. Non-parametric multivariate analyses of changes in community structure. Aust. J. Ecol., 18: 117-143.

Mezaki, T. and Kubota, K. 2012. Changes of hermatypic coral community in coastal sea area of Kochi, high-latitude, Japan. Aquabiology, 34: 332-337. (in Japanese with English abstract)

2) Books

Anderson, K. and Martin W. (ed.) 2009. "Distortion to agricultural incentives in Asia", The World Bank, Washington DC.

3) Parts of book

Huei, M.S., Chiu, L.I. and Men, C.Y. 1993. Mass mortality of prawn caused by Alexandrium tamarense blooming in a culture pond in southern Taiwan. In: Smayda, T.J. and Shimizu, Y. (eds.) "Toxic Phytoplankton Blooms in the Sea", Elsevier, New York, pp. 329-333.

4) Internet

FAO. 2005. National aquaculture sector overview- Cuba. http: //www. fao. org/fishery/countrysector/naso_cuba/en (Last access 7 March 2012).

10. Other instructions in making the reference list are as follows.

1) As for references written in languages other than English, give the English title and provide the information on the language and the presence of English abstract in parentheses at the end (see the second example of references); in this example, give "(in Japanese)" if the English abstract is absent. If the translated English title is not presented in the reference, give your own translation in brackets after the original title in Roman letters.

2) Thesis for a degree is referred to as a book, but give the comma-separated information of the kind of degree, the name of university or institution, and nationality, after the title of the thesis.

3) References in press (i.e., accepted for publication) should be listed with year to be issued and replacing unfixed issue and page numbers by "(in press)". If the year is not fixed, use "in press" instead of year.

11. Unpublished author's observations and the personal information that was directly provided to the author(s) should be indicated by "(unpublished data)" and "(personal communication)", respectively. The author must be responsible for citation of personal communications.

12. Internet information of which the uploaded year is unknown should be cited only in the text, not in a reference list, by listing the author (copyright owner), website name, URL, and last-access date.

13. In the text, two or more references within parentheses should be cited using a comma for a separator and in the same order as in the References section, such as (Kaufman 1994, Powles et al. 1999, Sy and Tsurufuji 1982a, b).

14. When citing figures or tables whose publication rights are assigned to other publishers, authors must state in the figure legends or table footnotes that permission from the publishers was obtained. Authors must also add the original articles from which they copy the figures or tables to the Reference list.

\section{Address for Editorial Correspondence}

Dr. Hiroshi Ueda, Editor-in-Chief of Kuroshio Science.

E-mail: hueda@kochi-u.ac.jp 